\title{
Multiple Response System: Evaluation of Policy Change in North Carolina's Child Welfare System
}

\author{
C. Nicole Lawrence *, Katie D. Rosanbalm, Kenneth A. Dodge \\ Center for Child and Family Policy, Duke University
}

\section{A R T I C L E I N F O}

\section{Article history:}

Received 25 May 2011

Received in revised form 9 August 2011

Accepted 10 August 2011

Available online 17 August 2011

\section{Keywords:}

Differential response

Alternative response

Child welfare reform

\begin{abstract}
A B S T R A C T
Systemic challenges within child welfare have prompted many states to explore new strategies aimed at protecting children while meeting the needs of families, but doing so within the confines of shrinking budgets. Differential Response has emerged as a promising practice for low or moderate risk cases of child maltreatment. This mixed methods evaluation explored various aspects of North Carolina's differential response system, known as the Multiple Response System (MRS), including: child safety, timeliness of response and case decision, frontloading of services, case distribution, implementation of Child and Family Teams, collaboration with community-based service providers and Shared Parenting. Utilizing Child Protective Services (CPS) administrative data, researchers found that compared to matched control counties, MRS: had a positive impact on child safety evidenced by a decline in the rates of substantiations and re-assessments; temporarily disrupted timeliness of response in pilot counties but had no effect on time to case decision; and increased the number of upfront services provided to families during assessment. Qualitative data collected through focus groups with providers and phone interviews with families provided important information on key MRS strategies, highlighting aspects that families and social workers like as well as identifying areas for improvement. This information is useful for continuous quality improvement efforts, particularly related to the development of training and technical assistance programs at the state and local level.
\end{abstract}

(c) 2011 Elsevier Ltd. All rights reserved.

\section{Introduction}

Over the past 20 years, child protective services (CPS) agencies have struggled with increasing numbers of child abuse and neglect reports, higher and more complex caseloads, and limited resources to meet the needs of families (Child Welfare Information Gateway, 2008; Shusterman, Hollinshead, Fluke, \& Yuan, 2005). These challenges prompted many states to develop new and innovative strategies aimed at addressing these problems, partly through the recognition that not all CPS reports require the same response or allocation of resources. One such strategy became known as differential response (DR). The foundational concepts underlying this secondary prevention model are flexibility, use of non-adversarial approaches, seeking to understand/identify root causes of maltreatment, and family engagement (Child Welfare Information Gateway, 2008; Conley, 2007). In this way, DR shifts the focus of CPS from a reactionary response to a particular incident, to one that assesses familial circumstances and assists families in addressing issues that impact child safety, with the goal of avoiding more serious maltreatment and/or future interaction with CPS. The growing interest in this model

\footnotetext{
* Corresponding author at: Center for Child and Family Policy, Duke University, Box 90539, Durham, NC 27708, United States. Tel.: +1 919668 3282; fax: +1 919668 6923.

E-mail address: nicole.lawrence@duke.edu (C.N. Lawrence).
}

among policy makers and child welfare administrators as well as the challenges inherent in implementing systems change has raised questions about how DR may impact child safety. The current study explored this issue and others, within the context of North Carolina's version of Differential Response.

Differential response is defined and implemented somewhat differently from state to state, but generally allows CPS agencies to respond to reports categorized as low or moderate risk using a noninvestigative assessment that typically does not require substantiation of abuse or neglect (Merkel-Holguin, Kaplan, \& Kwak, 2006). This approach focuses on assessing the family's strengths and needs and providing services and supports to address issues that are negatively affecting, or potential could affect, the safety and well-being of involved children. Factors such as the type and severity of abuse and neglect, previous involvement with CPS, and other risk factors are used at intake to determine the eligibility of reports for this non-investigative assessment, sometimes referred to as a family assessment.

In the mid-1990s, several states began implementing and evaluating differential response systems. In 2006, Merkel-Holguin and colleagues reported that 15 states had active differential response initiatives and three states had had previous initiatives no longer being implemented. Although numerous states have implemented various forms of differential response systems, few have approached this process as comprehensively as North Carolina. In addition to creating a differential response track, North Carolina developed a 
seven-part plan as part of their larger Multiple Response System (MRS) reform.

\subsection{MRS overview and history}

Two key influences served as the driving force behind the development of North Carolina's Multiple Response System (MRS): (1) the state's dual focus of ensuring safety, permanency, and nurturing homes for children, while also improving the lives of their families and (2) placement in federal program improvement as a result of the 2001 Federal Child and Family Services Review (Merkel-Holguin et al., 2006). The North Carolina General Assembly mandated that the state Division of Social Services develop and pilot a county-level system that uses a Family Assessment track (described below) for selected reports of child maltreatment, in addition to the traditional investigative process. The law also stipulated that data collection processes be established so that the state could assess the effects that the new system might have on child safety, timeliness of response, coordination of services, and cost effectiveness.

The North Carolina Department of Social Services chose 10 counties for a pilot implementation of MRS, purposely selecting for various sizes and geographic locations across the state. Selected counties were not provided additional funding to support this system change, however training and on-the-ground technical assistance were provided by NCDSS to ensure consistency in implementation and assist in the transition. The pilot project began in 2002. Based on favorable early reaction, in 2004, the legislature approved expanded implementation of MRS in an additional 42 counties. In 2006, the remaining 48 North Carolina counties began the implementation of MRS.

\subsection{MRS strategies: a family centered approach}

The Multiple Response System reform aims to increase family involvement in assessment and planning to address child welfare concerns and prevent future harm to children. The goal is to respond not only to the specific incident that brought a particular family to the attention of DSS, but to understand and address the broader spectrum of needs that might have undermined the caregivers' ability to parent effectively. Using a team approach, social workers work with the family to identify its needs and the available strengths and resources that will help family members improve their lives and better care for their children. The MRS assessment process aims to set a more cooperative tone and is designed to be more open and transparent than the traditional forensic assessment. The purpose is to engage the family and gain a more complete picture of their circumstances so that appropriate assistance can be offered and concerns remedied. When services are deemed necessary, the case planning process includes strategies to facilitate family participation and cooperation. When placement of children outside the home is required, MRS extends to the relationship between foster and birth parents, promoting interaction that supports a more seamless transition of childcare and reunification as soon as possible, when appropriate.

North Carolina's Multiple Response System policies outline seven key strategies for carrying out a family-centered approach to child protection, including:

\subsubsection{Choice between two approaches to reports of child abuse, neglect} or dependency

A choice in approaches allows for a differential response to child abuse, neglect and dependency reports, with options for the traditional investigative track or the new Family Assessment track. In North Carolina, the Family Assessment response is considered appropriate for reports meeting the statutory definitions of neglect, with the exception of abandonment and some special types of neglect reports. Neglect may include allegations of improper care, supervision or discipline as well as lack of medical care or injurious environments. North Carolina may assign less severe physical maltreatment cases, often categorized as "inappropriate discipline," to the family assessment track when appropriate. Similarly, other states such as Missouri, Minnesota and Ohio also allow such cases to be assigned to the differential response track. It is important to note that the choice of approaches may be used at the discretion of local CPS agencies; therefore, a report meeting the statutory definitions of neglect may still be assigned to the investigative track. The Family Assessment track provides a more tailored and holistic approach to working with individual families. The process engages families using a strengths-based model and facilitates a partnership among local agencies and communities to address all the needs of a child and family. Initial interviews of parents and children are scheduled with the parents, parents are informed about collateral interviews, and no perpetrator is identified. This track focuses on total child well-being, assessing all of the family's needs, rather than solely investigating a specific reported instance of maltreatment. The possible findings following a Family Assessment include: (a) Services Needed, indicating that child protective services are required (essentially, substantiation in the traditional system); (b) Services Recommended, indicating that services are voluntary but recommended; (c) Services Not Recommended, indicating that no service need has been identified; and (d) Services Provided (CPS no longer needed), indicating that appropriate services were provided during the assessment phase and CPS intervention is no longer necessary to ensure the safety of involved children. The traditional investigative track remains appropriate for allegations meeting the statutory definition of abuse, including substantial risk of serious injury to a juvenile by accidental or other means, cruelty, sexual abuse and moral turpitude.

1.2.2. Utilization of Child and Family Team meetings to make decisions

Child and Family Team (CFT) meetings aim to achieve safety, wellbeing and permanency for children and families by reaching out to family members (including extended family), natural family supports, and other community agencies. In doing so, CFTs encourage inclusion and active participation of these stakeholders in decision making and planning in all stages of the process from case management to foster care placement.

\subsubsection{Collaboration between Work First and the child welfare program}

Work First is the name under which North Carolina implements the Temporary Assistance to Needy Families (TANF) program. Collaboration between Work First and child welfare includes the coordination of joint home visits/case plans when possible, improved communication and information sharing, and inclusion of Work First personnel in CPS case staffings as well as CFT meetings as appropriate. This strategy was included to ensure that goals and case plans developed within these two agencies would complement rather than contradict one another.

\subsubsection{Implementation of a strengths-based, structured intake process}

This new process allows for the concerns of reporters to be heard, documented and screened using a highly structured intake tool that enhances the quality and consistency of information collected and emphasizes the strengths of the family as well as concerns and risk factors. Recognizing and documenting the strengths and cultural background of the families are considered paramount in the establishment of productive relationships and set the stage for the family-centered approach that is weaved throughout MRS strategies. This type of information is collected in addition to the typical intake information, enabling intake staff to determine if the allegations meet the statutory definitions of abuse, neglect, and/or dependency and should be accepted as a CPS report.

\subsubsection{Re-design of in-home services}

Re-structure of the case management system allows for more intensive services and contacts for families with more significant 
needs and less intensive or voluntary services to families with fewer needs or identified risks. Further, the re-design of in-home services emphasizes the engagement of families in the case planning/management process through CFT meetings as well as other mechanisms.

\subsubsection{Implementation of Shared Parenting meetings in placement cases}

Shared Parenting meetings bring birth parents and foster parents together as early as possible to encourage the development of ongoing interaction between the two. These meetings are meant to create a bridge between birth and foster parents for the purposes of easing the child's transition, enhancing the child's care, facilitating the mentoring of birth parents, and improving the chances of family reunification.

\subsubsection{Coordination between law enforcement agencies and child} protective services for the Investigative Assessment approach

Child protective services agencies developed formal Memoranda of Agreement with local law enforcement agencies to work in collaboration and share information in the investigation and prosecution of specific cases on the Investigative Assessment track.

\subsection{Evaluation of differential response}

Most studies of differential response systems have focused on specific states and have identified some positive effects on child safety/recidivism, family engagement and satisfaction, provision of services, and social worker satisfaction (Child Welfare Information Gateway, 2008; Zielewski \& Macomber, 2007). Among the most extensively studied state systems are Missouri, Minnesota and North Carolina (Waldfogel, 2009). A 2004 report by Loman and Siegel highlighted a quasi-experimental study conducted in Missouri from 1995 to 1998 which compared 14 small and medium-sized counties and selected zip codes within the St. Louis area implementing Family Assessment with 14 matched control counties/areas not implementing Family Assessment. Administrative data from the Missouri Division of Family Services in combination with family and social worker surveys and interviews, community provider surveys, and case file reviews revealed that Family Assessment did not compromise child safety, lowered rates of re-reports, did not affect rates of foster care placement, enhanced service delivery, increased family cooperation and satisfaction, and was viewed by social workers as a more effective approach. A follow-up study (Loman \& Siegel, 2004a) found that based on administrative data, repeat report rates in the 14 Family Assessment counties/areas continued to be lower 5 years after initial report. At the same time, this study revealed higher rates of subsequent out-of-home placements, with the greatest differences among the lowest risk cases and those with teenage children. The authors suggested that this effect may be due to an inadequate existing community service array to meet the needs of these families initially.

Loman and Siegel (2004b) also conducted a randomized-controlled trial of the alternative response demonstration project in Minnesota. In 2001,20 counties were selected to participate in the demonstration and were the subject of a comprehensive evaluation that included an impact and outcomes study, a process analysis, and a cost effectiveness study. The impact study included 14 counties where over 5000 families were screened in as eligible for alternative response and were randomly assigned to either the alternative response or to the control group receiving a traditional investigation. Analyses of administrative data revealed that families assigned to alternative response were less likely to have subsequent maltreatment reports as compared to the control group. Alternative response families also received more formal services apart from case management and were less likely to have children in out-of-home placements. The process study utilized feedback collected through surveys and interviews with nearly 1200 families, CPS staff, and other community stakeholders. Alternative response families were more likely to report that they were treated in a fair and friendly manner, social workers listened and tried to understand their situation and needs, the issues of importance to their family were discussed, and they were more involved in decision making. The majority of social workers held positive sentiments toward alternative response, with such attitudes tending to strengthen over time. Lastly, the results of the cost study showed that total costs for case management activities and other services were less for alternative response cases than control cases. More recently, Loman and Siegel (2010) conducted a replication of this evaluation design for Ohio's Differential Response System, yielding similar findings.

The only large-scale multi-state study utilized data from the National Child Abuse and Neglect Data System. Shusterman et al. (2005) compared children across six states (Kentucky, Minnesota, Missouri, New Jersey, Oklahoma, and Wyoming) who were referred to a differential response system with a non-experimental control group comprised of those receiving traditional investigations. Comparisons focused on case characteristics, circumstances of reports, and outcomes for children. The sample included approximately 314,000 children, of which 140,000 received a differential response during 2002. Findings revealed some consistencies with those obtained from the state-level evaluations. Across the six states, families receiving a differential response were more likely to receive services, suggesting to the authors that the less adversarial approach associated with differential response may make families more apt to engage and therefore benefit from services. In contrast to the state-level findings, this multi-state study found that recidivism rates for differential response cases did not differ from the rates for traditional investigative cases in any state except Oklahoma, where the recidivism rate was decreased. The authors conclude from this finding that children referred to differential response are not at greater risk for re-reports or future maltreatment.

One factor that may affect the success of differential response initiatives is the underlying assumption that if CPS identifies family needs, effective community services to address these needs are readily available (Zielewski \& Macomber, 2007). Where services are not adequate or accessible, CPS identification of family needs will not be sufficient to set families on a path toward better outcomes. This assumption may be of particular relevance in rural communities, as evidenced by a qualitative study by Zielewski and Macomber (2007) that examined service availability, service access and service networks associated with differential response systems in rural and urban communities within two states: Kentucky and Oklahoma. Researchers conducted focus groups and interviews with CPS staff, community-based service providers and families within two counties (one rural, one urban) in each of the states. The findings indicate that service availability in rural communities had few gaps, however, such communities tended to have less service capacity and fewer choices of providers. Service access was somewhat hampered in rural communities due to lack of transportation, the location of available services in proximity to where families reside and the associated travel time needed to participate in services. Urban areas had a different set of challenges around transportation such as inflexible schedules (i.e., no evening bus routes available) or difficulty utilizing sometimes complicated public transit systems.

In sum, evaluations suggest that differential response systems may reduce the likelihood of re-reports to CPS, can enhance service utilization by families, may reduce the likelihood of out-of-home placements, and can increase the level of family and social worker satisfaction. The current evaluation tested the impact and process of key strategies of MRS reform as implemented in North Carolina. The impact evaluation compared 9 of the counties that were selected for initial implementation of MRS with matched control counties across time with regard to:

- safety (rates of assessment, substantiation, and repeat assessment); - timeliness of response; 
- timeliness of case decision; and

- frontloading of services.

The process evaluation documented the implementation of MRS in the initial 10 counties, specifically:

- case distribution;

- implementation of Child and Family Teams;

- collaboration with Work First and other Community-based Providers; and

- Shared Parenting Activities.

\section{Impact evaluation of MRS}

The North Carolina Division of Social Services supported evaluation of MRS by an external evaluator to ensure that child safety is maintained, that families continue to receive timely response and needed services, and that local human services agencies are working together to accomplish these goals. Nine MRS counties were compared with 9 control counties selected from among the 48 counties that did not implement MRS until 2006. Each MRS county was matched to a control county based on total population, child population, past child maltreatment assessment rates, and past child maltreatment substantiation rates. The tenth MRS pilot county, Mecklenburg, could not be suitably matched due to its large population. To provide information on the comparability of the MRS and control counties, Table 1 shows the mean demographics for the 9 MRS and 9 control counties across the 6 years prior to MRS initiation, including both the variables used in matching counties and additional poverty-related variables.

\subsection{Methods and data sources}

The North Carolina Department of Health and Human Services collects data on each accepted CPS report of child maltreatment from each county. An "accepted" report is one which is determined through a structured intake process to be a credible allegation that should be processed further. Because data are not collected on non-accepted reports, the rate of non-accepted reports is not known. The data from accepted reports are entered into a central data registry maintained at the state level.

Data for the 9 pilot counties and 9 control counties analyzed in this study were extracted from the data registry for the period from July 1996 to December 2005 (prior to the start of MRS in control counties). Estimates of child population for each year in each county were obtained from the North Carolina Office of State Budget and Management website, which releases intercensal population estimates for July 1 each year. Data for children aged 0 to 17 were processed separately for each county to calculate unduplicated yearly and quarterly rates for three key outcomes: (1) maltreatment assessment rates per 1000 children,

Table 1

Comparison of MRS and control counties on baseline characteristics.

\begin{tabular}{|c|c|c|c|}
\hline & MRS county rate & $\begin{array}{l}\text { Control county } \\
\text { rate }\end{array}$ & t-statistic \\
\hline & $M(S D)$ & $M(S D)$ & \\
\hline Total population & $\begin{array}{l}122,367 \\
(115,099)\end{array}$ & $94,501(53,691)$ & $t(106)=1.6, n s$ \\
\hline Child population & $28,831(26,974)$ & $23,051(14,069)$ & $t(106)=1.4, n s$ \\
\hline $\begin{array}{l}\text { Maltreatment } \\
\text { assessment rate }\end{array}$ & $15.6(1.7)$ & $15.4(1.4)$ & $t(106)=0.5, n s$ \\
\hline $\begin{array}{l}\text { Maltreatment } \\
\text { substantiation rate }\end{array}$ & $5.0(0.5)$ & $4.8(0.5)$ & $t(106)=1.4, n s$ \\
\hline Median income & $\$ 35,821(3759)$ & $\$ 36,395(4286)$ & $t(88)=0.7, n s$ \\
\hline Unemployment rate & $4.1 \%(1.5)$ & $4.7 \%(1.6)$ & $\begin{array}{l}t(106)=1.9 \\
p=.06\end{array}$ \\
\hline $\begin{array}{l}\text { \% Children living } \\
\text { below poverty line }\end{array}$ & $18.8 \%(3.8)$ & $16.8 \%(4.2)$ & $t(88)=2.4, p=.02$ \\
\hline
\end{tabular}

(2) substantiated maltreatment rates per 1000 children, and (3) recidivism rates (i.e., unduplicated proportions of children investigated in a particular quarter who returned to CPS within 12 months for investigation of a new alleged event). After the implementation of MRS, numbers used in calculating substantiation rates include both traditional CPS substantiations and the MRS finding "Services Needed." These findings are both sufficiently serious as to require some action on the part of CPS, and thus are the most equivalent findings for comparing pre- and post-MRS maltreatment rates. For the purpose of clarity and simplicity, these will be referred to in combination as "substantiations" throughout this paper.

For summary data, weighting ensured that each county contributed equally within each analysis. Other data gathered from the central data registry included report and assessment dates (to monitor timeliness of response), case decision date (to monitor time to case decision), type of maltreatment reported, assessment track, case decision, and total minutes of services provided by CPS to each child.

\subsection{Analyses}

Regression-based interrupted time series (ITS) analyses were employed (Lewis-Beck, 1986). ITS analysis is a form of piecewise regression useful in examining time series data with fewer than 50 observations when there is some change in policy or procedures at a given time point. These models test for a change in either the mean level (i.e., intercept) or the slope of scores at the time of the policy change, or "interruption." The current models include a parameter for main effect of county type (MRS versus control) and interaction effects for county type by intercept and slope before the intervention and county type by change in intercept and slope after the intervention. The time-series regression equation is:

$$
\begin{aligned}
\mathrm{Y}_{1}= & b_{0}+b_{1} \mathrm{X}_{1 \mathrm{t}}+b_{2} \mathrm{X}_{2 \mathrm{t}}+b_{3} \mathrm{X}_{3 \mathrm{t}}+b_{4} \mathrm{X}_{4 \mathrm{t}}+b_{5} \mathrm{X}_{1 \mathrm{t}} \mathrm{X}_{4 \mathrm{t}}+b_{6} \mathrm{X}_{2 \mathrm{t}} \mathrm{X}_{4 \mathrm{t}} \\
& +b_{7} \mathrm{X}_{3 \mathrm{t}} \mathrm{X}_{4 \mathrm{t}}+\mathrm{e}_{\mathrm{t}}
\end{aligned}
$$

where $Y_{1}=$ the outcome variable (e.g., maltreatment substantiation rate per quarter); $\mathrm{X}_{1 \mathrm{t}}=\mathrm{a}$ counter for quarter, from 1 to $N ; \mathrm{X}_{2 \mathrm{t}}=\mathrm{a}$ dichotomous variable score 0 for observations before MRS and 1 for observations after MRS; $\mathrm{X}_{3 \mathrm{t}}=\mathrm{a}$ counter scored 0 for observations before MRS and ordinal from 1 to $N$ for each quarter after MRS; $\mathrm{X}_{4 \mathrm{t}}=$ county group (MRS versus control); $\mathrm{X}_{1 \mathrm{t}} \mathrm{X}_{4 \mathrm{t}}, \mathrm{X}_{2 \mathrm{t}} \mathrm{X}_{4 \mathrm{t}}$, and $\mathrm{X}_{3 \mathrm{t}} \mathrm{X}_{4 \mathrm{t}}=$ interaction of county group by each of the other variables; $e_{t}=$ error.

The parameter $b_{0}$ represents the intercept of the time series before implementation of MRS, whereas $b_{1}$ represents the pre-MRS slope. Parameters $b_{2}$ and $b_{3}$ represent changes in the intercept and slope of the time series, respectively, following MRS implementation. To evaluate changes as a result of MRS, we must examine the interaction effects by county type $\left(b_{4}\right): b_{5}$ represents the interaction between county type and pre-MRS slope, $b_{6}$ represents the interaction between county type and pre-post intercept change, and $b_{7}$ represents the interaction between county type and pre-post slope change.

Because MRS is a policy change that requires considerable shift in CPS worker philosophy and way of interacting with families, change might occur gradually following MRS implementation, resulting in a change in the trajectory of maltreatment rates rather than a sudden shift in mean. On the other hand, introduction of a new track and the use of new findings might cause an immediate shift in how cases are classified. With county condition (MRS versus control) included in the model, we compared intercept and slope changes between the MRS and control counties at the time of MRS implementation $\left(b_{6}\right.$ and $b_{7}$, respectively). Given the relatively small number of data points $(n=38)$, findings at both the statistically significant $(p<.05)$ and trend $(p<.10)$ level are reported.

To test for serial dependency and autocorrelation in the time series data, this procedure uses the Durbin-Watson statistic (DW; ideally approximately $=2$ ) and subsequently tests the rho ( $\rho$ : population 
correlation between error terms; ideally<.30) if the Durbin-Watson test suggests autocorrelation. If significant autocorrelation is found, a further step is taken to adjust for the autocorrelation in order to render the error terms independent.

\subsection{Projecting rate estimates}

In addition to examining statistical significance, we assessed the clinical significance of MRS by estimating how many children were prevented from involvement in a maltreatment assessment, substantiation, or repeat assessment following MRS initiation. Using data derived from ITS analyses, we projected what the rates of maltreatment assessment, substantiation, and repeats might have been in the absence of MRS. In other words, had MRS counties shown the same changes in intercept and slope as the control counties, what would maltreatment rates have looked like? To calculate projections, we used the regression equation generated by the ITS model with the post-MRS county-level interaction effects removed. This enabled us to estimate shifts for MRS counties equivalent to those seen in control counties. We then calculated the difference between observed and projected rates to estimate the real reduction in numbers of children assessed, substantiated, or re-assessed. Because the rates used in the regression were unduplicated only by quarter, a child can be counted more than once across quarters. To correct for this, we calculated the unduplicated numbers of children assessed, substantiated, and reassessed across the $31 / 2$ years prior to MRS. These numbers were compared with the numbers of assessments, substantiations, and reassessments calculated by adding the quarterly numbers across this same time period. The resulting ratio indicates the amount of duplication (children appearing in more than one quarter) during the $31 / 2$ years prior to MRS. Assuming the duplication rate would have remained constant following MRS, this ratio was applied to the summed quarterly post-MRS projected numbers to arrive at an estimate of the unduplicated number of children who would have had an assessment, substantiation, or re-assessment post-MRS in the absence of an intervention effect. Finally, we subtracted the actual unduplicated numbers post-MRS to produce an estimate of "prevented" assessments, substantiations, and repeat assessments.

\subsection{Results}

2.4.1. Pre-MRS comparison of pilot MRS counties and control counties

To ensure initial equivalence, MRS and control counties were compared on the variables used in matching: total population, child population, child maltreatment assessment rates, and child maltreatment substantiation rates prior to the initiation of MRS. Additionally, county-level information on median income, unemployment rates, and percent of children (aged 0 to 17) living below the poverty level were gathered from the Census Bureau and the KidsCount website to provide a richer contextual comparison of MRS and control counties. $T$-tests comparing rates for each of these variables during the years 1996 to 2001 found no significant differences between MRS and control counties on the variables used in matching, but there were small differences in unemployment and poverty rates (see Table 1). On average, unemployment rates were slightly lower in MRS counties, whereas child poverty rates were higher.

ITS models analyzed for maltreatment assessment and substantiation rates as part of the impact evaluation showed no main effect for county type (MRS versus control; see Table 2). This indicates that average baseline rates across all four matching variables were equivalent for MRS and control counties. A significant baseline difference in slope did emerge in the ITS model for substantiation rates, however; prior to MRS initiation, substantiation rates were increasing in MRS counties while remaining constant in control counties. Substantiation rate changes in MRS counties parallel the assessment rate changes, suggesting that the proportion of assessments
Table 2

Parameter estimates for child safety model effects.

\begin{tabular}{|c|c|c|c|}
\hline & Assessment rate & $\begin{array}{l}\text { Substantiation } \\
\text { rate }\end{array}$ & $\begin{array}{l}\text { 12-month repeat } \\
\text { assessment rate }\end{array}$ \\
\hline & $\beta(S E)$ & $\beta(S E)$ & $\beta(S E)$ \\
\hline Intercept & $13.6(0.4)^{* *}$ & $4.8(0.2)^{* *}$ & $23.9(0.6)^{* *}$ \\
\hline $\begin{array}{l}\text { Main effect } \\
\text { of county }\end{array}$ & $-0.5(0.6)$ & $-0.4(0.2)$ & $-0.2(0.9)$ \\
\hline Pre-MRS slope & $0.1(0.03)^{* *}$ & $-0.0(0.01)$ & $0.1(0.04)$ \\
\hline Intercept change & $-0.04(0.7)$ & $0.5(0.3)^{\wedge}$ & $0.3(1.0)$ \\
\hline Slope change & $-0.1(0.07)^{\wedge}$ & $-0.04(0.03)$ & $-0.1(0.1)$ \\
\hline $\begin{array}{l}\text { County } \times \text { pre-MRS } \\
\text { slope }\end{array}$ & $0.06(0.04)$ & $0.05(0.02)^{* *}$ & $0.1(0.06)^{*}$ \\
\hline $\begin{array}{l}\text { County } \times \text { intercept } \\
\text { change }\end{array}$ & $-0.4(1.0)$ & $-1.6(0.4)^{* *}$ & $-0.4(1.4)$ \\
\hline $\begin{array}{l}\text { County } \times \text { slope } \\
\text { change }\end{array}$ & $-0.1(0.1)$ & $-0.08(0.04)^{\wedge}$ & $-0.4(0.1)^{*}$ \\
\hline Overall $F$ statistic & $F(7,68)=18.7^{* *}$ & $F(7,68)=14.5^{* *}$ & $\mathrm{~F}(7,68)=7.4^{* *}$ \\
\hline $\begin{array}{l}\text { DW and } \\
\rho \text { statistics }\end{array}$ & $\begin{array}{l}\mathrm{DW}=2.0, \rho= \\
-0.01\end{array}$ & $\begin{array}{l}D W=1.7 \\
\rho=0.15\end{array}$ & $\mathrm{DW}=2.2, \rho=-0.1$ \\
\hline$R^{2}$ & 0.66 & 0.60 & 0.43 \\
\hline
\end{tabular}

$* * p<.01$.

$* p<.10$.

$p<.05$.

with substantiations was remaining constant for these counties. In contrast, the control counties showed stable substantiation rates despite increasing assessment rates; they substantiated a decreasing proportion of the assessments over time. This suggests possible policy differences in case finding decisions for MRS and control counties prior to MRS implementation. This will be considered in the discussion of findings.

\subsubsection{Child safety}

Safety was measured using rates of assessments, substantiated maltreatment (including findings of Services Needed), and repeat assessments. ITS analyses tested differences in level (intercept) and trajectory (slope) between MRS and control counties following MRS implementation, controlling for pre-MRS means and slopes. Figs. 1-3 illustrate findings from these analyses. Regression lines are shown by county group both before and after MRS initiation. Additionally, a dashed line indicates the projected rates that might have been seen in the absence of an intervention effect.

As depicted in Fig. 1, a trend-level main effect of slope was found for assessment rates, indicating declining slope after $2002(p=.09$, see Table 2). One possible explanation for this general decline in assessment rates beginning in mid-2002 is the introduction of the state-wide structured intake process, which provided clear guidelines for decisions on whether or not to follow up on a report with an assessment. Though MRS county assessment rates declined slightly

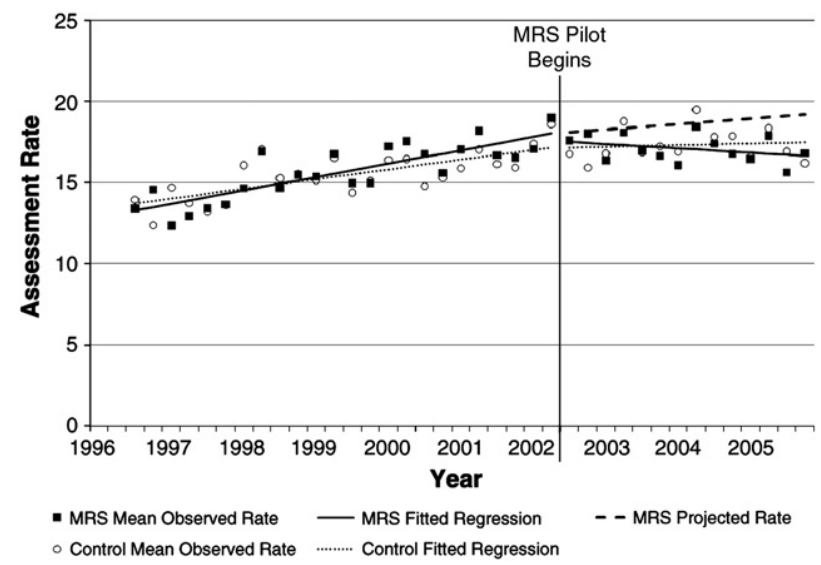

Fig. 1. Assessment rates per 1000 children age 0 to 17 : interrupted time series regression comparing MRS and control counties. 


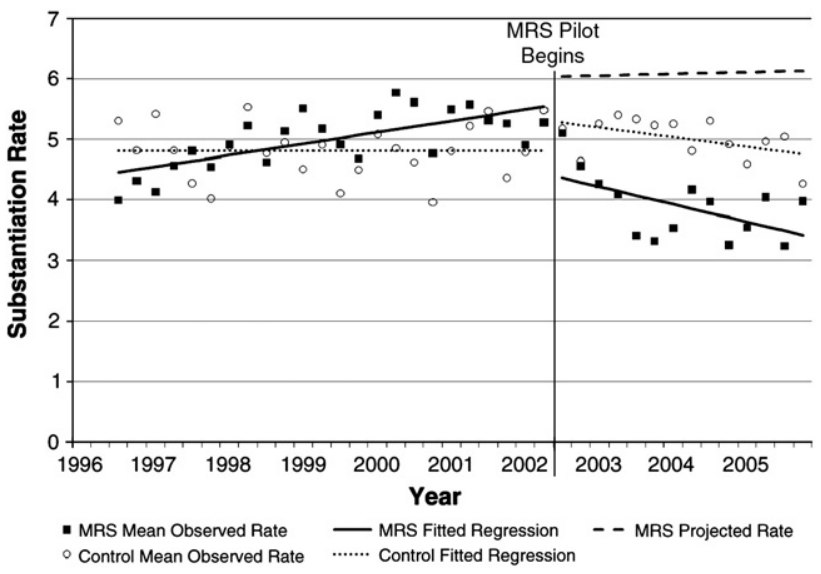

Fig. 2. Substantiation rates per 1000 children age 0 to 17: interrupted time series regression comparing MRS and control counties.

more than those of control counties, no significant interaction effects were found for the MRS-control comparison in intercept or slope. There is no evidence that the introduction of MRS altered the rate of assessment for maltreatment in MRS counties.

Fig. 2 depicts rates of substantiated maltreatment over time in MRS and control counties. Following the implementation of MRS, the mean rate of substantiated maltreatment dropped significantly in MRS counties compared with control counties $(t(1)=-4.0, p<.001$; Table 2). The change in rates occurred only in the MRS counties, and rates have continued to decline over time relative to rates in control counties (trend-level effect, $t(1)=-1.9, p=.06$ ). As mentioned previously, control counties were showing some differences in substantiation patterns prior to MRS implementation. Specifically, MRS counties maintained a consistent proportion of assessments that resulted in maltreatment substantiations, whereas control counties substantiated lower proportions of assessments over time. Given this pattern, it is even more striking that substantiation rates in MRS counties dropped to levels well below that of control counties.

For recidivism (repeat assessments, defined as the initiation of a new, independent assessment within 12 months of the previous assessment), the intercept did not change across counties, but the slope declined significantly in MRS counties relative to the control counties after MRS was introduced (Fig. $3 ; t(1)=-2.6, p<.05$ ). This finding indicates a favorable impact of MRS on the rate at which families returned for a new maltreatment assessment within 12 months of an earlier assessment.

Given that MRS showed significant effects on rates of substantiation and re-assessment, projected rates for these variables were

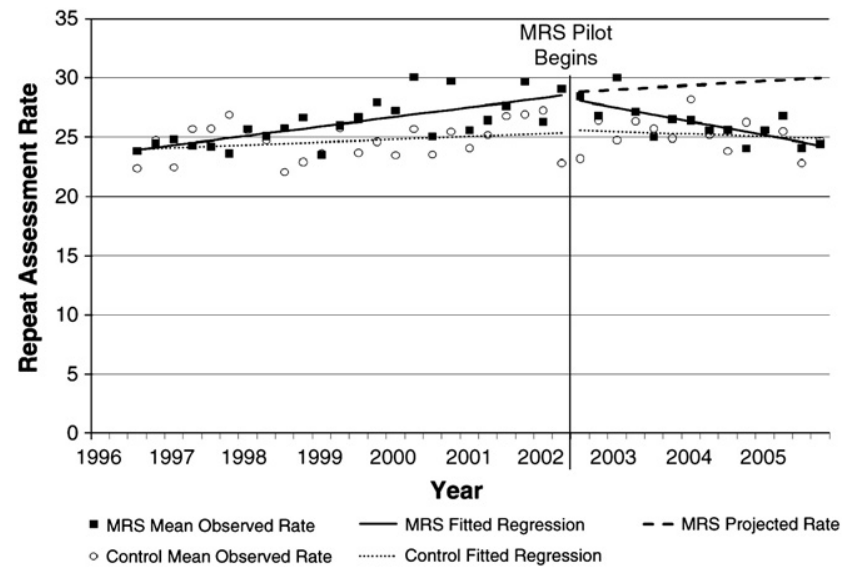

Fig. 3. Rate of repeat assessment within 12 months of initial assessment: interrupted time series regression comparing MRS and control counties. examined more closely to estimate clinically relevant prevention effects. Using the analyses described previously, we estimate that without the calculated effects of MRS, 6534 additional children ages 0-17 would have experienced a maltreatment substantiation in the nine MRS counties between mid-2002 and the end of 2005. Likewise, an additional 1149 children would have returned for a repeat assessment within 12 months of an earlier maltreatment assessment.

\subsubsection{Timeliness of response}

One concern with MRS implementation has been that changes in protocols will decrease the timeliness of initial case response and of overall assessment completion. Analyses addressed the proportion of cases for which an assessment began within the prescribed period of time and the proportion of cases for which case decisions were reached on time.

In North Carolina, County Departments of Social Services are required to initiate a response within a maximum of $72 \mathrm{~h}$ following receipt of an accepted report, with variable timelines dependent on the type and severity of allegation and the current level of risk to the child. A Priority Response Decision Tree is used for all accepted reports to determine if the required response time will be immediate, within $24 \mathrm{~h}$ or within a 72 -hour timeframe.

Analyses indicated that the proportion of cases meeting timeliness deadlines decreased at the trend level in MRS counties after MRS implementation as compared to control counties $(t(1)=-1.8$, $p=.08$; see Table 3 ), from an average of about $92 \%$ on-time responses to an average of $89 \%$ on-time responses. Control counties showed a smaller drop in on-time responses, falling from $94 \%$ to $93 \%$. By the end of 2005 , MRS counties returned to previous timeliness rates (92\%).

Before the introduction of MRS, all counties were required to complete investigations and to reach a case decision within 30 days from the report date. On August 1, 2002, a new policy was implemented for the Family Assessment track in MRS counties only. In order to allow social workers to put services in place during the assessment period without compromising child safety, the time frame for the completion of Family Assessments was extended to 45 days. Investigative Assessments were still to be completed within 30 days.

Though the trajectory of rates of on-time case decision differed marginally in MRS and control counties pre-MRS (with timeliness rates declining slightly in control counties), initiation of MRS did not alter the timeliness of case decision. MRS and control counties showed no significant differences in intercept or slope change for on-time case decisions following MRS implementation.

Table 3

Parameter estimates for timeliness and frontloading model effects.

\begin{tabular}{|c|c|c|c|}
\hline & Time to response & $\begin{array}{l}\text { Time to case } \\
\text { decision }\end{array}$ & $\begin{array}{l}\text { Frontloaded } \\
\text { services }\end{array}$ \\
\hline & $\beta(S E)$ & $\beta(S E)$ & $\beta(S E)$ \\
\hline Intercept & $96.2(0.5)^{* *}$ & $68.8(2.7)^{* *}$ & $432.6(15.6)^{* *}$ \\
\hline $\begin{array}{l}\text { Main effect } \\
\text { of county }\end{array}$ & $-4.9(0.7)^{* *}$ & $-2.2(3.8)$ & $-117.4(22.1)^{* *}$ \\
\hline Pre-MRS slope & $-0.1(0.03)$ & $-0.3(0.2)$ & $-2.7(3.1)$ \\
\hline Intercept change & $-1.3(0.8)$ & $-3.9(3.8)$ & $-0.2(17.2)$ \\
\hline Slope change & $0.2(0.1)^{* *}$ & $-0.3(0.5)$ & $6.5(3.4)^{\wedge}$ \\
\hline $\begin{array}{l}\text { County } \times \text { pre-MRS } \\
\text { slope }\end{array}$ & $0.1(0.05)^{*}$ & $0.5(0.3)^{\wedge}$ & $6.8(4.4)$ \\
\hline $\begin{array}{l}\text { County } \times \text { intercept } \\
\text { change }\end{array}$ & $-2.1(1.2)^{\wedge}$ & $5.9(5.5)$ & $43.1(24.3)^{\wedge}$ \\
\hline $\begin{array}{l}\text { County } \times \text { slope } \\
\text { change }\end{array}$ & $0.02(0.1)$ & $-0.6(0.6)$ & $-4.1(4.8)$ \\
\hline Overall $F$ statistic & $F(7,68)=28.1^{* *}$ & $F(7,68)=17.6^{* *}$ & $F(7,36)=29.4^{* *}$ \\
\hline DW and $\rho$ statistics & $\begin{array}{l}\text { DW }=1.9 \\
\rho=0.03\end{array}$ & $\begin{array}{l}D W=2.0 \\
\rho=0.02\end{array}$ & $\mathrm{DW}=2.1, \rho=-0.1$ \\
\hline$R^{2}$ & 0.74 & 0.45 & 0.85 \\
\hline
\end{tabular}




\subsubsection{Frontloading of services}

One of the major premises of MRS is that a family should be offered services as early as possible in the process to support their ability to keep their children safe and stable. Frontloading of services was defined as the number of minutes of CPS services (time that the CPS worker spent with the family or working on their case) provided subsequent to an accepted report of maltreatment and before a case decision was made, including both time spent in assessment activities as well as time in the facilitation of services from external agencies. Minutes of frontloaded services were not available electronically until 2000.

Fig. 4 depicts the mean number of minutes of frontloaded services provided for each case per quarter from fiscal year mid-2000 through end of 2005. MRS counties marginally increased the mean number of frontloaded minutes of service following MRS implementation relative to control counties (trend-level effect, $t(1)=1.8, p=.08$ ).

Given that frontloading is a goal of MRS, we also explored the effects of frontloading on child safety. Specifically, we used a logistic regression analysis to predict repeat assessment based on total number of frontloaded minutes. Time (pre-MRS versus post-MRS) and county group (MRS versus control) were included in the analyses, and all interactions were explored. Analyses showed that frontloading significantly decreased the probability that a child with an accepted report would return to CPS attention $\left(\chi^{2}(1)=19.6, p<.001\right)$. This pattern was equally true for both types of counties, but the effect was stronger post-MRS implementation (at the trend level, $\chi^{2}(1)=3.3$, $p=.07)$. In other words, families that received more frontloaded services during their assessment were less likely to be re-assessed for maltreatment in the next 12 months than were families that received fewer frontloaded services.

\subsection{Discussion}

In comparison with matched control counties, interrupted time series analyses revealed that the implementation of MRS was associated with: (a) lowered mean rates of substantiated maltreatment cases; (b) a shift in the trajectory of substantiation rates, such that rates began declining across time; and (c) a shift in the trajectory of repeat assessment rates, with a resulting decline in maltreatment re-assessment among those families that had been assessed previously. No differences were found in the overall rate at which cases were assessed for maltreatment. These findings indicate a favorable impact of MRS on child safety. In fact, analyses estimate that 6534 cases of substantiated maltreatment were prevented across the 9 MRS counties from mid-2002 through the end of 2005, as well as 1149 cases of repeat maltreatment assessments. Even if these estimates are overly optimistic, they still suggest the possibility of tremendous

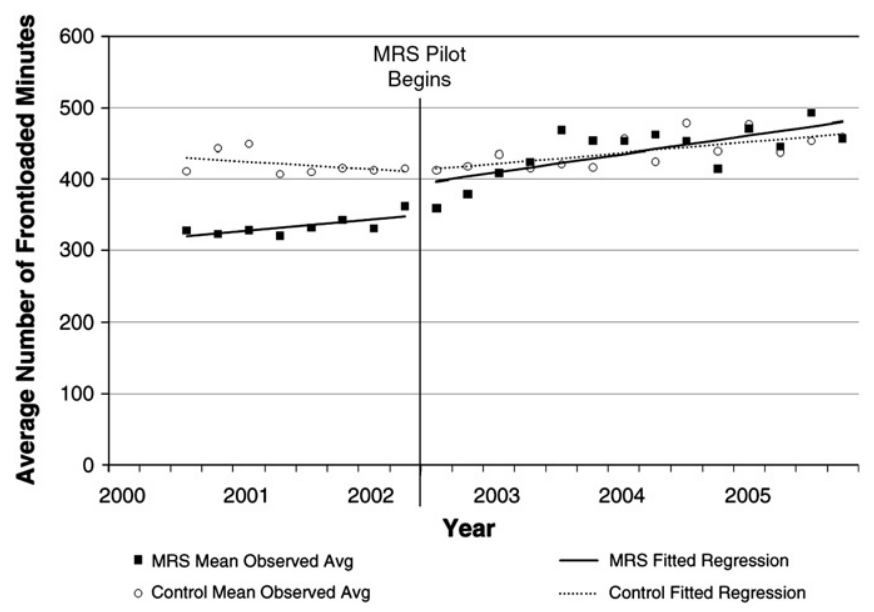

Fig. 4. Average minutes of frontloaded services: interrupted time series regression comparing MRS and control counties. benefits at the family and community levels over time, particularly now that all 100 North Carolina counties have adopted MRS.

Two important caveats temper these findings. First, evaluation of the impact of the North Carolina MRS was conducted by contrasting the mean and slope of county-level outcome variables for 9 counties that implemented MRS with those for 9 matched comparison counties, controlling for pre-MRS means and slopes. This method of evaluation is not as rigorous as random assignment; changes across time may be due to a time-varying county-level characteristic that is correlated with selection into MRS vs. control status (such as readiness for reform), rather than the MRS system. Second, the measure of substantiation in the family assessment track in MRS counties is a finding of Services Needed, whereas the measure for non-MRS counties is a traditional finding of substantiation of maltreatment. Although these outcomes convey similar meanings in the sense of child risk and need for family services, it is plausible that caseworkers implemented them differently. With added case finding options in MRS, caseworkers had more flexibility in assigning findings based on ongoing service needs rather than the facts of a specific incident. Cases that would previously have been substantiated may have had their needs met through frontloading during the assessment phase, thus prompting a finding of either Services Recommended (non-mandatory) or Services Received (no longer needed) at case decision. Nevertheless, substantiation rates showed more than just a shift in intercept that might be attributed to changes in case finding options; MRS counties also evidenced a shift in the slope of substantiation rates relative to control counties, with an ongoing decline in maltreatment substantiations. This is consistent with the increase in child safety suggested by the lowered rate of repeat assessment.

Timeliness of initiating assessment dropped in MRS counties relative to control counties after the implementation of MRS. From conversations with caseworkers, this temporary reduction in timeliness is related to the challenges of learning to juggle new types of caseloads, with more time spent providing comprehensive assessment and service referrals upfront. As staff and supervisors learned to adapt to the new system, workload was temporarily increased, making immediate response to new cases more challenging. The drop in on-time responses was minimal, however, and over time returned to previous levels. States planning differential response programs may want to consider this possible disruption in timeliness to initial contact and look for ways to diminish this effect as workers are adjusting to policy changes. Advance planning on prioritizing tasks within and across cases may be useful. States may also consider phasing in the differential response system by caseworker teams, allowing teams in transition to have temporarily lower caseloads as they adjust to new procedures.

In terms of time spent in the assessment process (time to case decision), MRS and control counties showed similar patterns over time. MRS counties were given additional time for Family Assessment cases, so the actual time to case decision is not reflected in this analysis, but proportion of cases completed within the mandated time frame was not affected by MRS.

In terms of actual services delivered, the implementation of MRS led to an increase in the frontloading of services, as measured by the number of minutes of services that each family received. For reasons that are not clear, control counties began with substantially higher levels of frontloading than did MRS counties. The implementation of MRS coincided with an average increase of $50 \mathrm{~min}$ of services per case, bringing MRS counties up to the level of control counties. The number of minutes of frontloaded services has continued to rise over time.

Increase in the quantity of frontloaded services appears to be a positive outcome for MRS, as families with a higher number of frontloaded minutes were found to have a lower rate of repeat assessment. This is a modest effect, but in contrast to what might be expected; one might expect caseworkers to spend more time with families who have higher needs, and these families might also be the ones most likely to return with a repeat report of maltreatment. The 
fact that analyses show just the opposite suggests that a focus on early efforts by social workers to complete a thorough assessment and link families with services is beneficial to the long-term well-being of families and children.

\section{Process evaluation in MRS counties}

Qualitative data from focus groups and caregiver telephone interviews were used to assess the quality of implementation related to a number of MRS strategies including: (a) collaboration/interface between CPS, Work First and other community-based providers, (b) Child and Family Team quality and impact, and (c) Shared Parenting Activities. These were strategies that cut across both the Investigative and Family Assessment tracks of MRS, thus families from both tracks were included in the sample.

\subsection{Methods}

\subsubsection{Focus groups and family phone interviews}

Thirty focus groups were facilitated in the 10 pilot counties. Each of the counties accommodated three separate focus groups that included social workers, social work supervisors and community partners/service providers. Each group was scheduled for approximately $1.5 \mathrm{~h}$ and was comprised of an average of 15 participants. The focus groups were digitally recorded and later transcribed. The transcriptions were then uploaded into Atlas.ti (qualitative software) in preparation for analysis. The transcripts were individually coded within Atlas.ti and reports were generated to identify themes across focus groups specific to key MRS strategies.

To gain additional perspective and to enhance continuous quality improvement in implementation, researchers conducted telephone interviews with 223 caregivers. Agency staff members in the pilot counties were asked to collect consent forms and contact information from caregivers willing to share their recent experience with DSS in a confidential telephone interview. Caregivers were at various points along the CPS service continuum at the time of the interview, including the investigative or family assessment phase, case management/inhome services or foster care. The sample included a mix of cases that were on the traditional investigative track as well as the Family Assessment Track. A total of 479 consents were received and of those, 256 were not completed for various reasons including disconnected or wrong numbers, inability to make contact, respondent refusal to participate, or incorrect/inappropriate respondent (e.g., contact information was for kinship care providers). The remainder were successfully contacted and participated in the survey. During the 15- to 30minute telephone interview, caregivers were asked about their involvement with DSS, including how the social worker treated them, what services they received, whether their ideas were incorporated into plans, whether the help they received improved their parenting, overall level of understanding about MRS and satisfaction with the interaction. These data were entered into a database and descriptive statistics were generated to explore aspects of MRS implementation from the perspective of caregivers.

The information obtained through the caregiver phone interview was not intended to provide summative conclusions regarding the effectiveness of MRS, but rather served as a useful tool for improving the implementation of MRS. It is important to note that findings should be interpreted cautiously and with limitations of these data in mind. Self selection bias was likely a factor because families were asked by their social workers to voluntarily sign the consent if they had an interest in participating. Families in crises or those with the most severe CPS cases or familial challenges may not be fully represented within the sample. Further, because social workers collected the consents, it is possible that they did not ask families to participate with whom they had contentious relationships, fearing that families would provide negative feedback. Finally, social workers did not collect data specific to the number of families that refused to sign an informed consent and as such, there is not reliable way to determine how many families chose not to participate or other case specifics about those who refused.

Table 4 highlights select demographics for respondents participating in the caregiver phone interviews. Approximately a third of the sample were married (28.2\%), one third were single (33.6\%) and the remaining third were divorced, widowed or separated (33.5\%). The sample was closely split between African American and White respondents at $46 \%$ and $43 \%$ respectively. Hispanic/Latino respondents made up only $5 \%$ of the sample, with the remaining $5 \%$ categorized as "other." Two thirds of the sample (60\%) had not completed HS or had a HS Diploma/GED. Almost half the respondents (47.5\%) were unemployed and 64.1\% indicated that their annual household income was less than $\$ 25,000$. Those earning $\$ 35,000$ or more per year made up only $14 \%$ of the total sample. Roughly half of all families surveyed said that they had had previous involvement with CPS.

\subsection{Results}

\subsubsection{Case distribution}

In 2002, the MRS pilot counties implemented both the dual response approach to assessments (Family Assessment vs. Investigative Assessment) and a new system of case decisions for the Family Assessment Track (Services Needed, Services Recommended, Services Provided, and Services Not Recommended). The Investigative Assessment track continued to use the pre-existing case decision system (Substantiated vs. Unsubstantiated). We descriptively examined changes in the usage of assessment tracks and case findings across the years of MRS implementation to gain a better understanding of how MRS is used in practice.

In the first year of MRS implementation, fewer than 40\% of all CPS cases were handled using family assessment. This rate jumped to $70 \%$ in the second year as counties grew more familiar and comfortable with the new track, and continued rising to near $80 \%$ in 2008. In terms of case findings, the rate of Services Needed and Services Provided (CPS no longer needed) remained relatively constant over time, but use of Services Recommended and Services Not Recommended findings changed considerably in the first year of implementation. Services Recommended increased from just under $20 \%$ to just over $30 \%$ of all family assessment findings, whereas Services not Recommended dropped from $65 \%$ to near 50\%. Growth in the Services Recommended category is an ideal outcome as workers continue to master the tenets of family-centered practice and expand the numbers of families who participate in voluntary services.

Table 4

Respondent demographics - caregiver telephone interview.

\begin{tabular}{lc}
\hline Demographics & $\%$ of respondents \\
\hline Characteristics & \\
Marital status & \\
$\quad$ Married & $28.2 \%$ \\
Single & $33.6 \%$ \\
Divorced/Widowed & $17.0 \%$ \\
Separated & $16.5 \%$ \\
Race/Ethnicity & \\
African American & $46.1 \%$ \\
White & $43.0 \%$ \\
Hispanic & $5.3 \%$ \\
Other & $5.3 \%$ \\
Education levels & \\
Less than HS & $31.8 \%$ \\
HS/GED & $28.2 \%$ \\
Some college & $34.9 \%$ \\
College degree & $4.9 \%$ \\
\hline
\end{tabular}




\subsubsection{Implementation of child and family teams}

Data related to the implementation of CFT meetings were acquired through provider focus groups and caregiver telephone interviews. Focus group participants offered numerous examples of positive experiences and good outcomes related to CFT meetings, with particular attention to the benefits associated with holding such meetings. Overall, participants reported that CFT meetings improve social work practice because they provide a unified forum for problem solving, with the family as a central participant. The benefits of CFT meeting as described by providers included:

- Improved communication and trust with families, who come to see the team as a support system rather than a group of accusers;

- Enhanced transparency of the process, leading to better interagency collaboration; and

- Improved case plan development, resulting in higher levels of adherence and better outcomes.

Participants also described a number of barriers associated with the implementation of CFTs, with two key themes emerging: difficulty in managing the practical logistics of CFT meetings and family preparation. The challenges described by providers included:

- Difficulty accommodating the schedules of both families and community partners;

- Low levels of participation by community partners due to low school personnel availability, inability of some service providers to bill for time spent in CFT meetings, the "after hours" time frames of many meetings, and inter-agency conflicts;

- Lack of dedicated facilitators to support and manage the process; and

- Lack of family preparation, engagement and understanding of the process.

In order to explore how CFT meetings are being implemented from the perspective of the family, the caregiver phone interview included a series of questions on this topic. This represented a challenge in the administration process because many families were not clear as to whether they had been involved in a CFT or other similar meeting. Interviewers provided an explanation as to what a CFT is and some examples of who might have attended such a meeting, but still many respondents could not confirm having participated.

Of the 223 phone interview respondents, only 60 indicated that they had a CFT or similar meeting. It is important to note that about half (118) of the families were in the Investigative or Family Assessment phase of their cases at the time of interview, meaning that they typically would not have had an opportunity to participate in a CFT because their case had not yet progressed to case management or in-home services where such meetings are typically facilitated. Still, this finding supports provider concerns that families were not sufficiently prepared for CFTs and did not understand the process.

A key aspect of CFT meetings that adhere to model fidelity is the early formation of a team that is inclusive of extended family members, other natural supports and community-based service providers. With this team in place, when CPS is no longer involved with a family, there remains a team to provide needed supports and avoid repeated involvement by CPS. Families who reported on CFT participation noted that apart from themselves, the other attendees of CFT meetings were grandparents and other extended family (45\%), children (28\%), GAL representatives (10\%), social workers $(100 \%)$, social work supervisors (75\%), foster parents (8\%) and other agency representatives or service providers (33\%). This finding suggests that many CFT meetings had an array of participants, but fewer than half had extended family and other natural supports.

Another important aspect of CFT model fidelity is shared power and decision making, which includes: informing families about the purpose and processes associated with CFT meetings; allowing the family to determine who will be part of their team; and encouraging high levels of family engagement/participation. More than half (58\%) of families who reported having a CFT said that they felt they had a say in who was invited to come to the meeting and were encouraged to bring supports and other family members. More than $60 \%$ indicated that the purpose of the meeting was explained to them clearly. Over $80 \%$ indicated that they were comfortable or somewhat comfortable sharing their ideas during the meeting. Despite a high degree of comfort sharing ideas, more than 50\% felt their ideas were not taken seriously and were not included in the resulting case plan.

\subsubsection{Shared Parenting Activities}

Information about the implementation of Shared Parenting was collected through focus groups and caregiver phone interviews. Focus group discussions centered on the perceived effectiveness of this strategy and barriers to implementation. Overall, participants expressed positive attitudes about Shared Parenting as a strategy for engaging both foster parents and birth parents. It was suggested that these meetings can be highly useful in achieving numerous desired results for foster care cases by effectively:

- Easing the transition and associated anxiety for children and birth parents;

- Facilitating long-lasting relationships between birth parents and foster parents, often leading to the provision of respite care and/or on-going support for birth parents after the children return home; and - Reducing time to reunification in some cases.

Amid the positive comments expressed about Shared Parenting, a number of barriers to implementation were also discussed. The barriers mentioned focused on three key themes:

- Foster parent resistance related to the desire to adopt and/or difficulties overcoming the familial circumstances that placed the children in care;

- Birth parent resistance due to anger about the removal of their children and/or denial around the issues that created the safety risk; and

- Seven-day time frame for implementation of Shared Parenting meetings creating logistical challenges and impacting the "readiness" of foster parents and birth parents to engage in the process.

The caregiver phone interview asked respondents who had children in foster care a series of questions about their experiences. Only $13 \%$ of the 223 families participating in the phone interview (including Investigative and Family Assessment cases) indicated that they had a child in foster care at the time the survey was administered. All of these respondents indicated that their social workers had helped them to stay in contact with their children through arranging visits, assisting with transportation needs or some other assistance; $32 \%$ of respondents said they believed they had input in decisions made about their children while in foster care; and $57 \%$ of respondents indicated that they recalled participating in a Shared Parenting meeting. All who had participated in Shared Parenting meetings said they were encouraged to share information about their child's everyday routines, and the majority (53\%) believed that their ideas and comments were taken seriously.

\subsubsection{Collaboration with Work First}

Collaboration between CPS and Work First is one of the seven core strategies of MRS and important in avoiding duplication of services. Data regarding the level of collaboration between the two entities were collected through provider focus groups and caregiver phone interviews. Focus group discussions centered on: (a) the use of Work First staff as collateral contacts, (b) the processes associated with determining dual involvement, and (c) the development of joint case plans. The following points highlight the findings:

- Social workers and supervisors indicated that they currently use Work First staff as collateral contacts when they are aware of common clients; 
- The process by which they become aware of common clients varied by county, but in most cases CPS intake workers were responsible for determining if a family has involvement with other county services;

- The majority of social workers and Work First representatives noted that they engage in some form of case coordination to ensure efforts are not being duplicated; and

- The volume of cases simultaneously involved with CPS and Work First is not high.

There were some indications that joint home visits including both Work First representatives and CPS social workers have occurred in several counties, but it was clear that this practice is not a common occurrence. Social workers commented that the goals of CPS home visits and Work First visits are often too different and cannot always be appropriately combined. Overall, these findings suggest that whereas there are few cases with simultaneous Work First and CPS involvement, more often than not, the two programs are aware of each other's involvement and work to coordinate case management activities.

The caregiver phone interviews supported the focus group findings by asking families a series of questions about dual involvement with CPS and Work First. The results showed that only $8 \%$ of respondents had both a CPS and Work First case open simultaneously: $4 \%$ had a Work First case prior to their CPS case, with $4 \%$ becoming involved with Work First subsequent to the opening of a CPS case. Few (2\%) indicated that they had participated in a joint meeting with both their CPS and Work First workers.

\subsubsection{Collaboration with other community-based providers}

Focus group discussions related to the nature of the collaboration between county divisions of social services and other agencies or community-based organizations included broad representation from various organizations. The discussions focused on two topic areas: the level of inter-agency collaboration and concerns. Discussions about inter-agency collaboration yielded overwhelmingly positive comments related to how effectively DSS is engaging other agencies to meet the needs of families. The following themes emerged from these discussions:

- DSS agencies have developed strong partnerships with community partners;

- Social workers and supervisors are generally viewed in a positive light; and

- There seems to be a high degree of interaction among and between agencies.

While the majority of community partners expressed positive views about their working relationships with county DSS agencies, a number of concerns were also noted. The concerns expressed tended to focus on a few topic areas, including:

- The belief that DSS is not holding families accountable or is not doing enough to protect children with the implementation of MRS;

- Lack of feedback or follow-up specific to reports made by community partners;

- The need for greater clarity and consistency regarding what casespecific information DSS can share with community partners and what cannot be shared; and

- Challenges associated with building on-going relationships with social workers due to high levels of staff turnover.

\subsubsection{Family feedback}

Data about the experiences of families involved with CPS were obtained through the caregiver phone interview. Families were asked to share their opinions about their overall interaction with CPS, the effectiveness of services provided in helping them with various aspects of family functioning, and what they might change about the way CPS works with families in the future. When asked to describe their feelings about their overall experience with CPS, 65\% of the comments were coded as positive (e.g., hopeful, relieved, pleased, satisfied, or happy), with $27 \%$ categorized as negative feelings (e.g., angry, stressed, annoyed, or worried), and $8 \%$ provided more neutral responses. Of the families interviewed that had received services/assistance through CPS $(n=94)$, roughly $31 \%$ reported that the services improved their parenting skills; $28 \%$ said that it helped them to better deal with conflict; $21 \%$ said it helped them to know who to contact in the community should they need assistance in the future; $32 \%$ said it helped them to better provide for their family's needs; and 1\% said it helped them to feel better about themselves and their family. The fact that a relatively small proportion of families who received services indicated that such services improved family functioning within these domains may suggest that there are not enough appropriate, high quality services or providers available within the surveyed counties, that many families did not value the services they received, or that they didn't believe they needed them in the first place.

When respondents were asked what they would change, if anything, about the way that CPS works with families, $24 \%$ of families noted that they would not change anything. Seventy-six percent offered suggestions for improvements which centered on improving communication and respect, reserving judgment, and retaining the same social worker for the duration of the CPS case.

\section{Conclusions}

MRS, North Carolina's differential response system, was gradually phased in across 4 years, allowing for evaluation of early process and outcome effects. This plan was purposeful on the part of the North Carolina legislature, primarily to ensure that the option of a Family Assessment track for certain types of lower-risk cases would not adversely affect child safety. This plan also provided the opportunity for a feasibility study, as ten pilot counties worked out the challenges associated with the new system and made adjustments to make the program work within the confines of tight budgets and staffing. It is important to note that MRS counties were not provided additional funding to support this systems change but, rather, were asked to embrace a paradigm shift in social work practice that in many ways challenged some of the traditional approaches to working with families involved with CPS. In fact, this cost-neutral approach may actually have the potential to reduce the costs associated with protecting abused and neglected children by reducing the likelihood of repeat assessments.

This paper reviewed the primary outcomes examined across the first 4 years of MRS implementation, comparing pilot counties to control counties similar in child population and maltreatment rates. During the first year of MRS implementation, the new Family Assessment track was used for fewer than $40 \%$ of the CPS cases. In subsequent years, pilot counties settled into a rate of case assignment to the Family Assessment track that was between 70 and $80 \%$. This is on the higher end of the range as compared to differential response systems in other states, which have tended to assign $42 \%$ to $71 \%$ of cases to the alternative track (Shusterman et al., 2005). Similarly, it took about a year for the Services Recommended finding to be used fully, but after a year it leveled off at around 30\% of Family Assessment findings.

Assessment rates decreased somewhat following MRS implementation, but not significantly more for MRS pilot than for control counties. Substantiation rates declined significantly more for MRS than for control counties and continued to decline more sharply over the four years following MRS implementation. This effect could be a function of the change in case options, particularly initially, but the continued trend suggests that children are no less safe than before MRS, and may even be safer. The findings for recidivism support this conclusion. MRS counties showed a declining slope for repeat assessments relative to control counties, meaning that their repeat assessment rates fell more steeply 
after MRS was implemented than did those of control counties. These figures suggest that MRS may be improving child safety by meeting families' needs at a level sufficient to keep them from returning to CPS with maltreatment concerns. This finding is consistent with those of other states using differential response systems with moderate risk families (e.g., Loman \& Siegel, 2004a, 2004b).

As a measure of increased time spent working directly with families in the assessment phase, frontloading of services was quantified as the number of minutes that a worker spent in billable client assessment or service activities prior to the case decision. MRS is meant to enhance the provision of services during the assessment phase, with the goal of meeting as many family needs as possible up front, and ideally moving the family to a place where they no longer require CPS involvement to maintain safety. Though it was only a trend effect, MRS pilot counties did appear to increase service frontloading relative to control counties. This is an important effect, as frontloading was also found to predict family safety as measured by likelihood of repeat assessment. Families with more minutes of social worker services prior to case decision were less likely to return with a maltreatment allegation within the next 12 months. This correlation held for both MRS pilot and control counties, suggesting that an emphasis on comprehensive assessment and early intervention can have long-term benefits for families.

Overall, MRS has met with success in North Carolina, with positive effects for child safety and minimal impacts on timeliness. In addition, families and social workers report that MRS is serving to improve rapport and family engagement. Findings from the focus groups and caregiver interviews suggest that Child and Family Teams, the MRS strategy at the core of family-centered practice, can be an effective tool in engaging families, informal supports and community partners when: meetings are inclusive of various stakeholders and natural supports; families are appropriately prepared; family ideas are incorporated into resulting plans; and barriers to implementation are strategically addressed. Similarly, Shared Parenting meetings are thought to help forge relationships between foster parents and birth parents and ease the transition into care for children when: they are implemented consistently; issues contributing to resistance on either side are identified and addressed; and families are encouraged to share information about their children and are engaged in the process.

Data collected specific to the level of collaboration occurring between DSS and Work First showed that whereas cases with simultaneous involvement of both government programs were relatively few, social workers are utilizing their Work First counterparts as collateral contacts and are engaging in joint case planning and/or coordination in situations where there are mutual clients. It also appears that DSS is effectively collaborating with community partnering agencies, but there are areas that, if addressed, could foster improvements. For example, the establishment of processes or mechanisms for providing a feedback loop on common cases would help to ensure that strong collaborative relationships endure even with high levels of social worker turnover. Providers at community agencies also expressed some concerns that families involved with MRS lacked sufficient accountability, possibly affecting child safety. This finding suggests a need for community outreach and education about MRS. Evaluative findings to date have not found children to be less safe due to the implementation of MRS, so it is possible that misconceptions or lack of information about MRS policy may be contributing to these perceptions. Outreach efforts were conducted in the pilot counties early in the implementation process, but they should be part of an ongoing effort to educate stakeholders about this evolving systems change.

Family perspectives about their experiences with CPS as gauged through the caregiver phone interview showed that overall, families expressed many more positive feelings about their interaction with
CPS than negative. This pattern could be an early indicator that negative perceptions about the role of CPS in the community are starting to change as a result of direct interactions with social workers. This idea is further supported by the fact that nearly a quarter of respondents indicated that they would not change anything about the way that CPS works with families, though again, self selection may cause bias in the results of these interviews.

Amid these positive findings, it is important to consider that of those families that received services through CPS, a significant proportion did not find such services to be particularly useful in improving family functioning. It is not clear as to why many of the families found services to be less valuable, but it may be an indicator of poor service array/ quality or that families did not agree they needed the services included in their case plan. Further exploration of this issue is warranted given the emphasis on providing these resources to families and the associated costs to communities to provide them.

In conclusion, findings from the impact evaluation provide clear evidence that the use of differential response in assessing suspected maltreatment does not decrease child safety, and indeed appears to increase safety. Information from the process evaluation provides useful feedback on ways to improve MRS in North Carolina and in similar differential response systems in other states. Given that the observed benefits were achieved without any additional funding, continued implementation and ongoing quality improvement are clearly worthwhile. With both encouraging child safety outcomes and positive reactions from social workers and families, differential response systems appear to be a favorable change for child welfare.

\section{Acknowledgments}

This work was supported by an evaluation grant to the Center for Child and Family Policy at Duke University from the North Carolina Department of Health and Human Services. Points of view or opinions in this paper are those of the authors and do not necessarily represent the official position or policy of the sponsor. Author Dodge acknowledges the support of Senior Scientist Award 2K05DA015226.

\section{References}

Child Welfare Information Gateway (2008). Differential response to reports of child abuse and neglect. Issue brief. Washington, DC: U.S. Department of Health and Human Services, Administration for Children and Families.

Conley, A. (2007). Differential response: A critical examination of a secondary prevention model. Children and Youth Services Review, 29, 1454-1468, doi: 10.1016/j.childyouth2007.06.003.

Lewis-Beck, M. S. (1986). Interrupted time series. In W. D. Berry, \& M. S. Lewis-Beck (Eds.), New tools for social scientists: Advances and applications in research methods (pp. 209-230). Beverly Hills, CA: Sage.

Loman, L. A., \& Siegel, G. L. (2004). Differential response in Missouri after five years, final report. St. Louis, MI: Institute of Applied Research.

Loman, L. A., \& Siegel, G. L. (2004). Minnesota alternative response evaluation, final report. St. Louis, MI: Institute of Applied Research.

Loman, L. A., \& Siegel, G. L. (2010). Ohio alternative response pilot project evaluation: Final report. St. Louis, MI: Institute of Applied Research.

Merkel-Holguin, L., Kaplan, C., \& Kwak, A. (2006). National study on differential response in child welfare. Denver, CO: American Humane Association and Child Welfare League of America.

Shusterman, G. R., Hollinshead, D., Fluke, J. D., \& Yuan, Y. T. (2005). Alternative response to child maltreatment: Findings from NCANDS. Washington, DC: U.S. Department of Health and Human Services, Office of the Assistant Secretary for Planning and Evaluation. Retrieved March 10, 2010 from http://aspe.hhs.gov/hsp/05/childmaltreat-resp/.

Waldfogel, J. (2009). Differential response. In K. A. Dodge, \& D. L. Coleman (Eds.), Preventing child maltreatment, community approaches (pp. 139-155). New York, NY: The Guilford Press.

Zielewski, E. H., \& Macomber, J. E. (2007). Rural families' connections to services in an alternative response system. Journal of Public Child Welfare, 1(4), 21-41, doi: $10.1080 / 15548730802118215$. 